\title{
CLINICAL AND ECONOMIC BURDEN OF INVASIVE MENINGOCOCCAL DISEASE IN GERMANY
}

Liping Huang', Denise Hever ${ }^{2}$, Jan Multmeier ${ }^{3}$, Sabrina Janßen ${ }^{3}$, Dennis Häckl ${ }^{4}$, Niklas Schmedt ${ }^{2}$

' Pfizer Inc., Collegeville, PA, USA | ${ }^{2}$ Institute for Applied Health Research Berlin, Berlin, Germany I 3 Pfizer Deutschland GmbH, Berlin, Germany I 4 WIG2 GmbH, Leipzig, Germany

\section{BACKGROUND}

Invasive meningococcal disease (IMD) is an unpredictable and life threatening disease.

- IMD is associated with substantial morbidity and mortality with rates of $\sim 10 \%$, and $\sim 20 \%$ of survivors are affected by permanent complications 1

Although IMD is part of the infectious disease surveillance in Germany, limited data is available on detailed clinical

characteristics, long-term outcomes, health care resource utilization (HRU) or the attributable costs of IMD.

\section{OBJECTIVES}

To examine short and long-term clinical characteristics of IMD patients, HRU and related costs in Germany.

\section{METHODS}

Data source: InGef (Institute for Applied Health Research Berlin) database which includes longitudinal inpatient and outpatient claims data of statutory health insurance providers in Germany2.

Study design: A retrospective cohort study based on claims data

Inclusion criteria: First hospitalization with IMD (ICD-10 GM code A39) as main discharge diagnosis between 2009 and 2015 (admission date=index date) (Figure 1). And continuous enrollment of at least one year or from birth onwards (average follow up $=48.5$ months $(S D=28.7$ months)

Figure 1 Schematic illustration of the IMD cohort

INDEXX DATE: HOSPPLZATION FOR IMD

BASEINE PEPOOO

ENROLMENT PERIOO WTH MAX. FOLLW.UP. 7 YEARS END OF STUOY PERROO

Statistical analysis method:

Descriptive analyses were conducted. For HRU and costs: IMD cohort was matched by age and sex with a non-IMD cohort (1:4 matching). All IMD cases $(N=18 ; 11 \%)$ and matched controls $(N=99 ; 15 \%)$ with extremely high cost in baseline were excluded. Mann-Whitney Utest was used to test the difference of costs between IMD cases and matched controls

\section{LIMITATIONS}

Due to the low number of IMD cases, the statistical power was low.

Costs obtained for this study only include direct medical costs from the perspective of German SHls, but no indirect costs.

IMD by serogroups were not distinguished

\section{RESULTS}

Figure 2: Age/sex and baseline risk condition, and the distribution of type of IMD (N= 164 IMD patients)

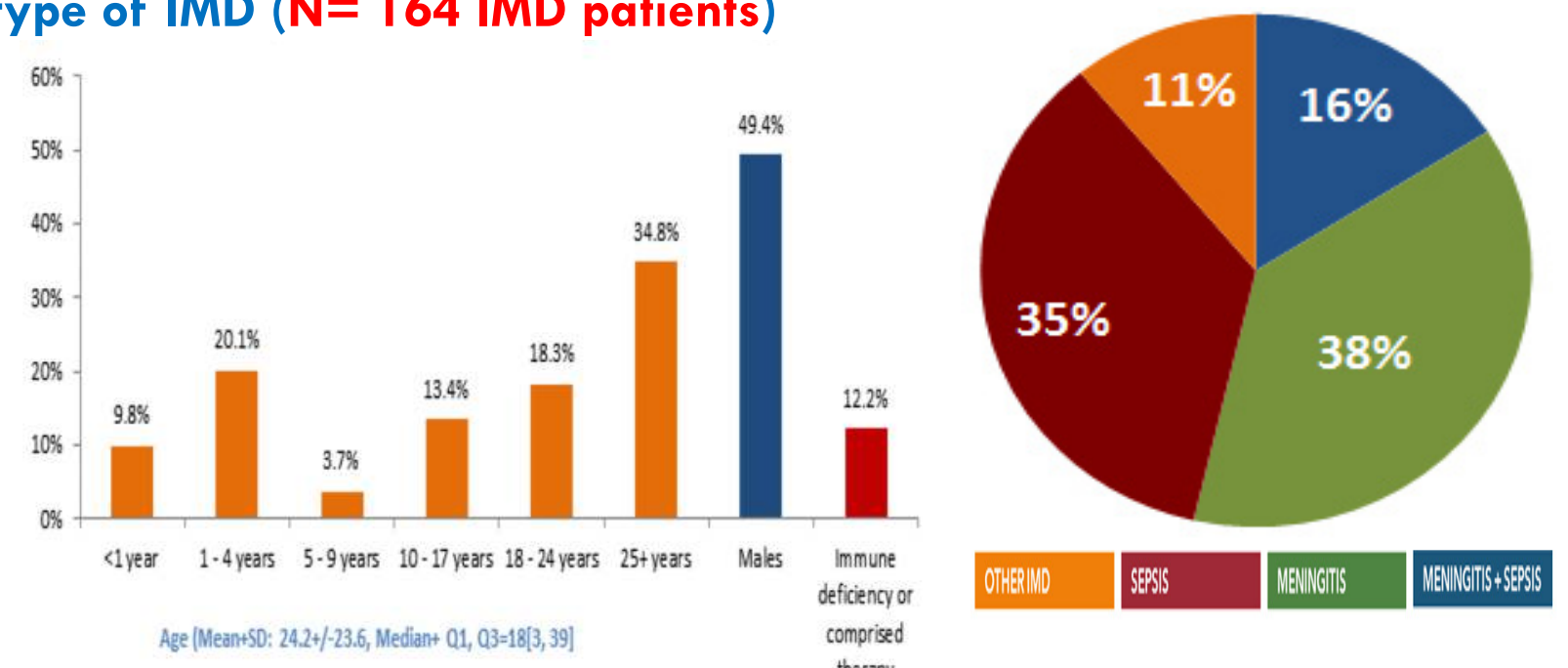

Figure 3: Clinical Outcomes of IMD Cases

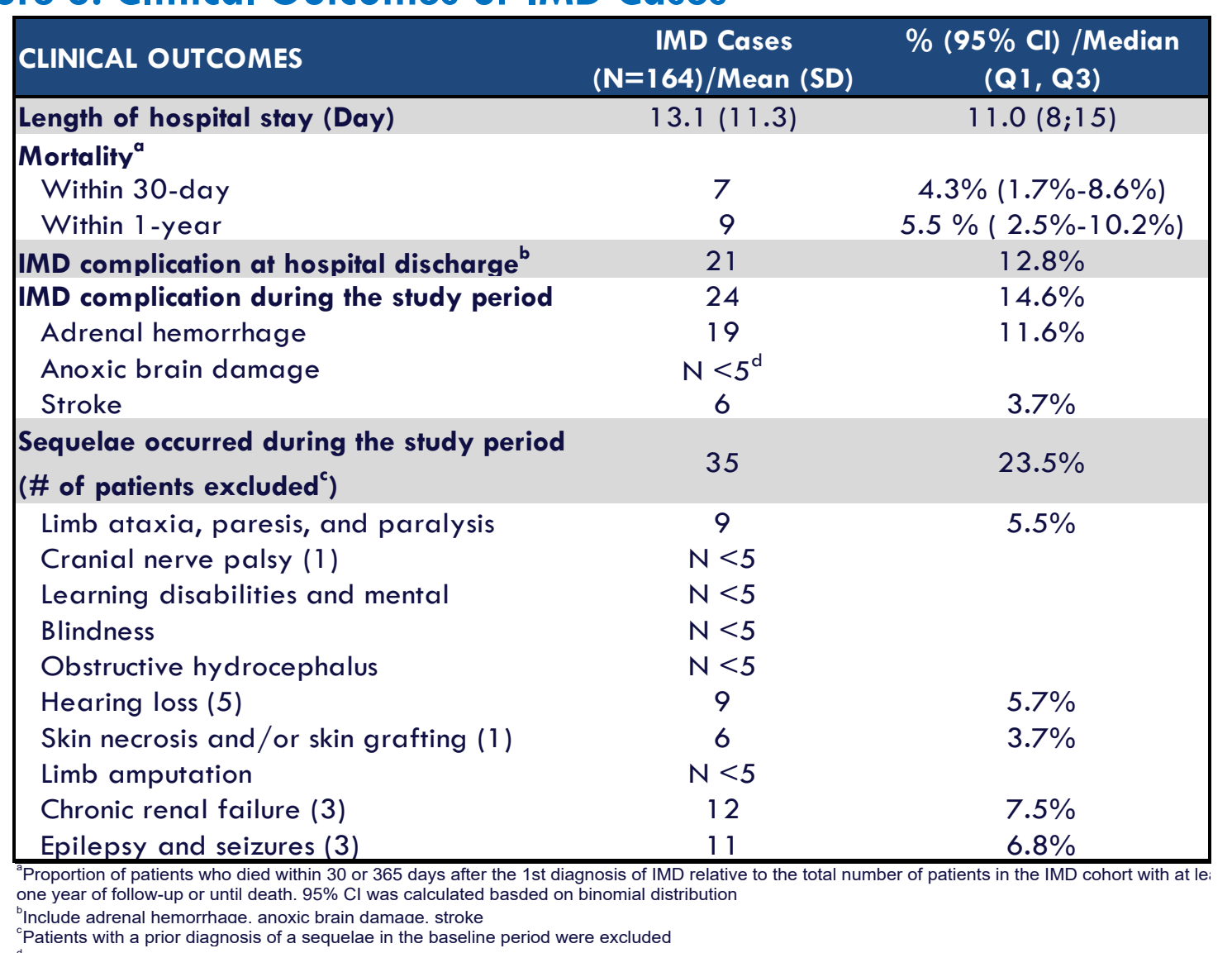

Figure 4: Mean overall actual costs within pre-defined time periods

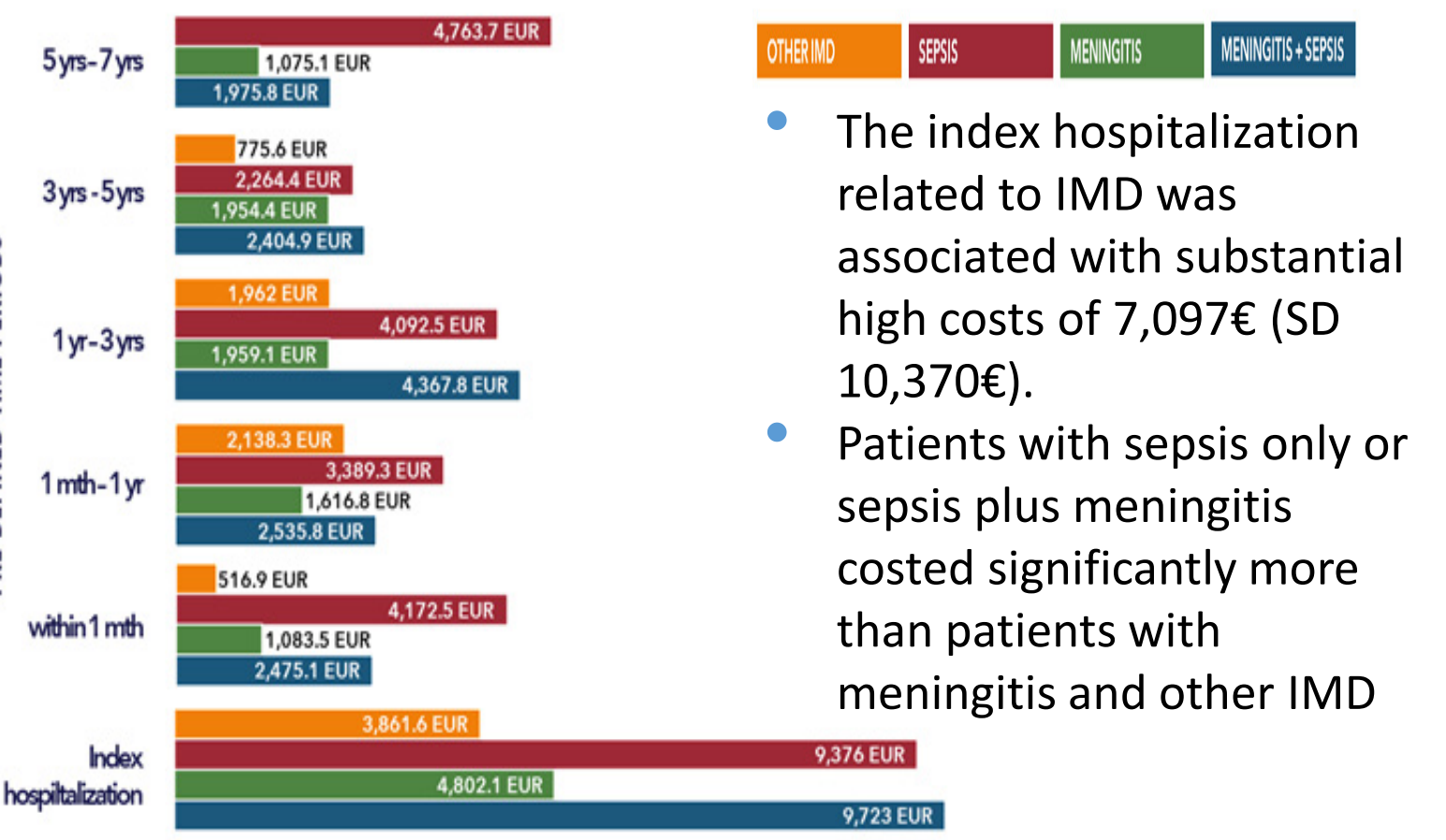

\section{RESULTS (Cont.)}

Figure 5: Comparison of overall actual mean costs of IMD cohort and matched Non-IMD cohort within pre-defined time periods

\begin{tabular}{|c|c|c|c|c|}
\hline & IMD MATCHED & MATCHED NON-IMD & SMD & P.VALUE \\
\hline Zaseline (N) & 146 & 557 & & \\
\hline Mean (SD) & $€ 644$ ( €815) & $€ 718$ (€995) & 0.08 & 0.982 \\
\hline Within 1 month & 146 & 557 & & \\
\hline Mean (SD) & $€ 2,277(€ 19,462)$ & $€ 50(€ 162)$ & 0.25 & $<0.001$ \\
\hline 1 month to 1 year & 138 & 553 & & \\
\hline Mean (SD) & $€ 1,954(€ 5,830)$ & $€ 885(€ 2,372)$ & 0.32 & $<0.001$ \\
\hline 1 year to 3 years & 130 & 520 & & \\
\hline Mean (SD) & $€ 2,791(€ 7,474)$ & $€ 1,767(€ 3,547)$ & 0.22 & 0.700 \\
\hline 3 years to 5 years & 96 & 390 & & \\
\hline Mean (SD) & $€ 1,834(€ 3,833)$ & $€ 1,436(€ 2,906)$ & 0.13 & 0.426 \\
\hline 5 years to 7 years & 56 & 208 & & \\
\hline Mean (SD) & $€ 2,523(€ 6,195)$ & $€ 1,682(€ 4,194)$ & 0.18 & 0.298 \\
\hline
\end{tabular}

SMD=Standardized mean difference
Resulted from Mann-Whitnev U-test

Figure 6: Comparison of mean costs of HRU between IMD and non-IMD

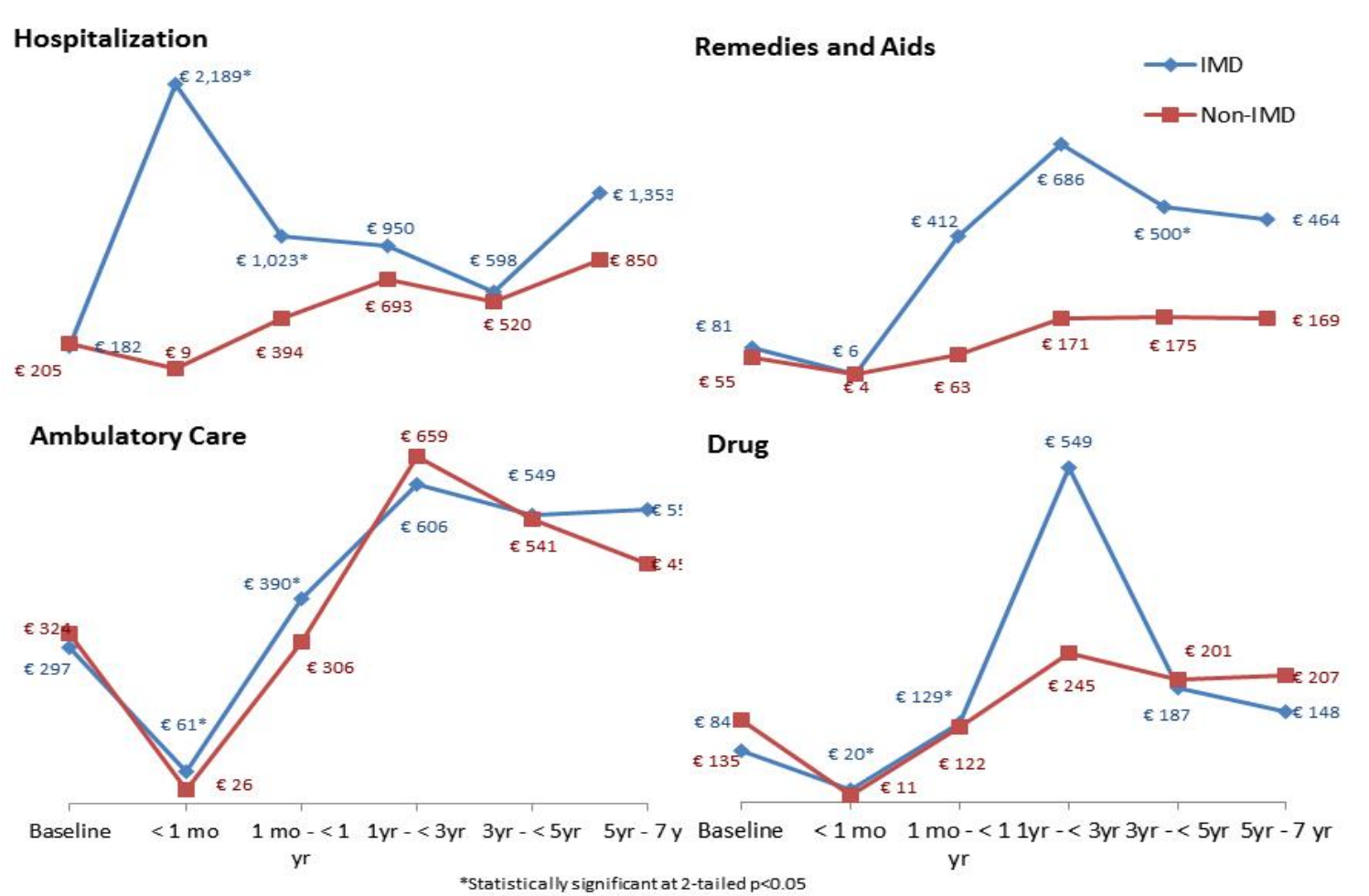

CONCLUSIONS

- This study highlighted the cost, especially cost during the first month of IMD and underlines the importance of preventive measures against IMD such as vaccinations.

- Further studies with larger sample sizes of IMD cases are required to estimate the long-term effects of IMD with larger statistical precision.

\section{REFERENCES}

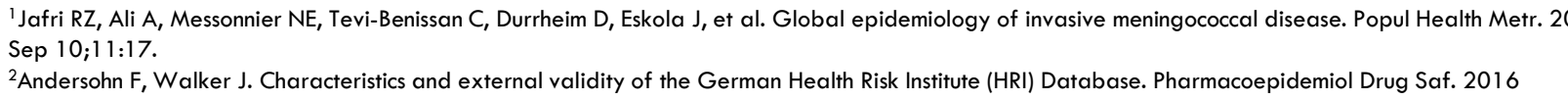

